\title{
Situación y avances en la poscosecha y procesamiento del aguacate (Persea americana Mill.)
}

\section{Avocado (Persea americana Mill.) postharvest status, breakthrough and processing}

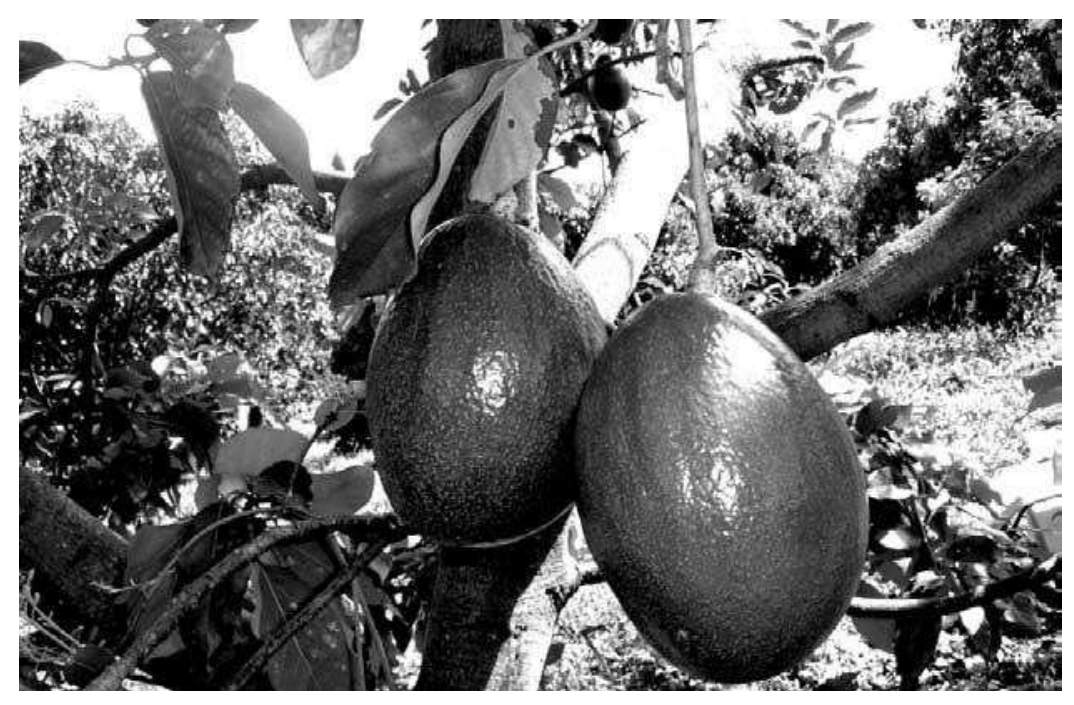

FREDDY FORERO1

JAIRO GARCÍA ${ }^{2}$

JULIÁN F. CÁRDENAS-HERNÁNDEZ

Frutos de aguacate cerca a su madurez fisiológica.

Foto: F. Forero

\section{RESUMEN}

El aguacate, es una fruta muy apetecida en el mercado mundial por su consistencia, exquisito sabor, alto valor nutritivo y amplias posibilidades de uso no solo en culinaria sino en procesos cosméticos y farmacéuticos. A pesar de esto, los avances tecnológicos que se producen en la cadena productiva del aguacate, no son divulgados y transferidos de una forma efectiva a los productores y comercializadores. En el presente artículo se recopilan y analizan investigaciones que han sido reportadas en fuentes bibliográficas especializadas; en el área de poscosecha se tratan los temas de almacenamiento refrigerado, regulación de etileno, atmósferas modificadas y control de microorganismos, que son las técnicas más utilizadas para mantener la calidad de esta fruta en fresco. En el procesamiento del aguacate se presentan las diversas alternativas tecnológicas que se han desarrollado para la conservación y aprovechamiento de la pulpa, aceite y semilla, igualmente una descripción de los usos y perspectivas que tienen estas materias primas en el desarrollo de productos no tradicionales.

1 Investigador, Centro de Investigación Nataima, Corporación Colombiana de Investigación Agropecuaria (Corpoica), Grupo Interdisciplinario de Investigación en Frutales, Espinal (Colombia). forero@corpoica.org.co

2 Investigador, Centro de Investigación Nataima, Corporación Colombiana de Investigación Agropecuaria (Corpoica), Grupo Interdisciplinario de Investigación en Frutales, Espinal (Colombia).jgarcia@corpoica.org.co

3 Estudiante Ingeniería Agronómica, Facultad de Ciencias Agropecuarias, Grupo de Investigación Ecofisiología Vegetal, Universidad Pedagógica y Tecnológica de Colombia, Tunja (Colombia).julianc0182@yahoo.es 


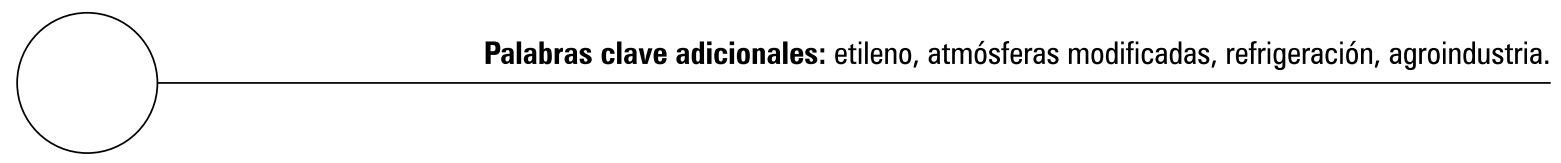

\section{ABSTRACT}

Avocado is a very wanted fruit worldwide for its consistency, exquisite taste, high nutritional value and its wide potential use not just for cooking, but also in cosmetic and pharmaceutical processes. Despite this, the technological advances developed in the productive chain of avocado, are nor spread and transferred in an effective way to producers and dealers. In the current article are collected and analyzed studies that have been reported in specialized literature, in the postharvest area. Topics as refrigerated storage, ethylene regulation, modified atmospheres and microorganisms control, were discussed being the most applied techniques to maintain the fresh quality of this fruit. In the avocado processing are shown some developed technological alternatives used for the conservation and utilization of pulp, oil and seed, also a description of the uses and perspectives that have these raw materials in the development of non traditional products.

Additional key words: ethylene, modified atmospheres, refrigeration, agroindustry.

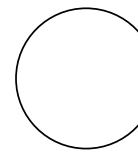

INTRODUCCIÓN

El aguacate (Persea americana Mill.), es una fruta apreciada en el mercado mundial por su consistencia suave similar a la de la mantequilla, su exquisito sabor, su alto valor nutritivo y por sus amplias posibilidades de uso no solo en la culinaria sino también en procesos agroindustriales (Olaeta, 2003). Se estima que actualmente existen más de 500 variedades, sin embargo, por razones de productividad y otras del mercado que van desde su perecibilidad hasta su contenido graso, la explotación comercial se limita a un número muy reducido de estas (Avilan, 1994).

El área mundial cosechada ascendió a 416.287 ha con producción de 3.187.534 taaño-1 en 2004. Sosteniendo un permanente incremento en su producción mundial, pasando de producirse 2.089.894 $t$ en 1994 a 3.187.534 t en 2004. Las importaciones mundiales presentaron un crecimiento anual entre 1994 y 2003 del 9,02\%, los mayores importadores de aguacate han sido principalmente los Estados Unidos y Francia, el primero, con $32 \%$ del total de las importaciones mundiales, y el segundo con el 20\% (Agexpront, 2004).

Por otra parte, existe la opinión de que la industria aguacatera es muy pequeña, pero con grandes oportunidades de desarrollo sobre todo con la diversificación de mercados y la presentación final del producto, es decir, no limitarse únicamente a la venta de producto en fresco, sino también enfocar esfuerzos a la comercialización de productos procesados. Tiene gran aceptación y con un mercado doméstico fuerte, con amplias oportunidades de participación en los mercados internacionales, tanto en fresco como procesado en guacamole, puré, aceite comestible, jabones, cosméticos y chamú, entre otros (Asohofrucol, 2006). 
La exportación en fresco a los Estados Unidos está creciendo en forma importante, al ampliarse las regiones exportadoras autorizadas e incrementarse los estados de ese país a los que se permite su importación (APHIS, 2004). Aunque Estados Unidos registra importaciones durante todo el año, existe una marcada estacionalidad, dado que entre septiembre y diciembre se concentra el $80 \%$ de las importaciones globales, alcanzando un pico (25\%) en el mes de noviembre, justo cuando se inicia la temporada (marketing season) en ese país (Today's Market Prices, 2003).

\section{POSCOSECHA}

La calidad del aguacate se relaciona con diversos factores y puede tener distintos significados en las diferentes etapas de la manipulación de la fruta en poscosecha. El consumidor percibe la calidad en términos de apariencia, sabor y precio. Por otro lado, el manipulador de la planta de clasificación y embalaje, el distribuidor y minorista pueden percibir la calidad en términos de la facilidad de manipulación, uniformidad de embalaje y ausencia de defectos físicos, fisiológicos y patológicos.

En cambio, el productor puede percibir la calidad como la ausencia total de defectos, una óptima distribución de tamaños y la distribución general de calibres. Tradicionalmente se ha puesto poca atención a la optimización de la calidad de la fruta, tanto en tiempo de cosecha como a lo largo de su manipulación hasta que llega al consumidor. En Colombia estos controles de calidad están reglamentados por el Instituto Colombiano de Normas Técnicas, bajo las normas NTC 1284 (Icontec, 1994), NTC 1248-2, NTC 1248-3 (Icontec, 1996), NTC 5209 (Icontec, 2003), que establecen las definiciones, condiciones de cosecha y almacenamiento, requisitos mínimos de calidad, madurez, clasificación, disposiciones referentes al calibre, tolerancias y presentación para las variedades Booth 8, Choquette, Fuerte, Hass, Lorena, Trapp, Trinidad y Santana. Una relación de los parámetros de calidad se muestra en la tabla 1.

Es básico recordar que el aguacate es un fruto climatérico (Seymour et al., 1993); en segundo lugar, es muy susceptible al daño por frío (Woolf et al., 2004), lo que significa que durante el almacenamiento el fruto podría sufrir fácilmente una descomposición fisiológica. Pero se pueden realizar muchos manejos que mitigan estos daños. Woolf et al. (1995) observaron una mayor tolerancia del aguacate a bajas temperaturas después de tratamientos con aire caliente, de hasta $34^{\circ} \mathrm{C}$. Estos tratamientos además redujeron la tasa de maduración. Tales efectos también

Tabla 1. Características físicas promedio, para ocho variedades de aguacate en Colombia.

\begin{tabular}{|l|r|r|r|l|c|c|}
\hline \multicolumn{1}{|c|}{ Variedad } & $\begin{array}{c}\text { Longitud } \\
(\mathrm{mm})\end{array}$ & $\begin{array}{c}\text { Diámetro } \\
(\mathrm{mm})\end{array}$ & $\begin{array}{c}\text { Relación } \\
(\mathrm{L} / \mathrm{D})\end{array}$ & \multicolumn{1}{|c|}{ Forma } & $\begin{array}{c}\text { Peso } \\
(\mathrm{g})\end{array}$ & $\begin{array}{c}\text { Cáscara } \\
(\mathrm{mm})\end{array}$ \\
\hline Hass & 88,6 & 66,4 & 1,3 & Ovoide & 197,0 & 1,45 \\
\hline Fuerte & 119,5 & 76,2 & 1,6 & Piriforme & 334,1 & 0,84 \\
\hline Booth 8 & 106,8 & 84,9 & 1,3 & Ovoide & 387,4 & 1,41 \\
\hline Trinidad & 99,4 & 90,1 & 1,1 & Esférico & 410,2 & 0,72 \\
\hline Lorena & 128,9 & 94,5 & 1,5 & Piriforme & 457,6 & 0,85 \\
\hline Trapp & 137,4 & 94,5 & 1,5 & Piriforme & 552,2 & 1,11 \\
\hline Choquette & 130,5 & 99,2 & 1,3 & Ovoide & 662,4 & 1,53 \\
\hline Santana & 159,7 & 97,1 & 1,6 & Piriforme & 683,4 & 1,41 \\
\hline
\end{tabular}

Fuente: Rojas et al., 2004 
habían sido observados en fruta expuesta a altas temperaturas (exposición al sol) sobre el árbol (Woolf et al., 1999; 2000).

Algunos trabajos han buscado identificar las variables que más influencian la duración del aguacate una vez colectado: se ha encontrado que los factores fecha de cosecha, período de almacenamiento en frío y temperatura de almacenamiento, provocan las variaciones más significativas en los parámetros de calidad (Bravo, 1997). El régimen de temperatura recomendado debe ser visto como una herramienta flexible en el manejo de poscosecha, ya que la reacción de la fruta al almacenaje en frío puede diferir de temporada a temporada, y si no se maneja adecuadamente puede causar gran variedad de defectos externos (Arpaia et al., 2004), entre los más recientemente reportados se encuentra el daño a las lenticelas del aguacate, que se manifiesta como puntos marrón, causados por el cambio de volumen que sufre el mesocarpio de la fruta durante un mal almacenamiento refrigerado, lo cual provoca la ruptura de las lenticelas y su posterior pardeamiento (Everett et al., 2007).

\section{Regulación del etileno}

El control de etileno en ambientes de almacenamiento juega un papel principal en la prolongación de la vida de muchos productos frescos (Saltveit, 1999). Sin embargo, durante los últimos años la investigación de materiales nuevos y más eficaces para la depuración de etileno ha sido escasa. Por otro lado, en el aguacate ha habido un incremento exponencial en la investigación del inhibidor de etileno 1-Metilciclopropeno (1-MCP) (Feng et al., 2000; Jeong et al., 2002; Matthew et al., 2005; Watkins, 2006).

Pesis et al. (2002) demostraron que la aplicación exógena de etileno, sin importar el método, causa una intensificación en la decoloración del aguacate almacenado en refrigeración, también afirma que el tratamiento con Etrel $^{\circledR}$ (Ácido 2-cloroetil fosfónico) previo al empaque, causa seve-
Tabla 2. Parámetros mínimos de calidad, para ocho variedades de aguacate en Colombia.

\begin{tabular}{|l|c|c|c|c|} 
Variedad & $\begin{array}{c}\text { Pulpa } \\
(\%)\end{array}$ & $\begin{array}{c}\text { Aceite } \\
(\%)\end{array}$ & $\begin{array}{c}\text { Materia } \\
\text { seca } \\
(\%)\end{array}$ & $\begin{array}{c}\text { Dureza } \\
\left(\mathrm{kg} \cdot \mathrm{cm}^{2}\right)\end{array}$ \\
\hline Hass & 55,7 & 25,5 & 39,5 & 2,4 \\
\hline Fuerte & 70,6 & 10,0 & 21,1 & 2,2 \\
\hline Booth 8 & 69,2 & 6,4 & 15,8 & 3,2 \\
\hline Trinidad & 62,9 & 10,8 & 23,9 & 2,4 \\
\hline Lorena & 69,2 & 3,3 & 15,9 & 3,7 \\
\hline Trapp & 68,4 & 3,8 & 14,9 & 4,8 \\
\hline Choquette & 77,2 & 4,6 & 14,8 & 2,7 \\
\hline Santana & 68,6 & 3,9 & 12,0 & 4,1 \\
\hline
\end{tabular}

Fuente: Rojas et al., 2004

ros síntomas de daños por frío, de igual forma corroboran la acción benéfica que tiene el 1-MCP sobre el retraso de la maduración de aguacates de las variedades 'Hass' y 'Fuerte', que fueron aptos para el consumo después de 3 semanas de almacenamiento a $5^{\circ} \mathrm{C}$, lo cual atribuyen a la reducción significativa de la actividad para la enzima polifenoloxidasa (PPO), que es una de las mayores responsables del pardeamiento en el aguacate.

Se ha buscado combinar la efectividad del 1-MCP, con otro tipo de productos que produzcan beneficios adicionales, tal como lo ensayaron Jeong et al. (2003), quienes aplicaron el tratamiento con 1-MCP y luego cubrieron los aguacates (Tower II y Booth 7) con cera (Sta-Fresh $819^{\circledR}$ ), a fin de reducir la pérdida de agua y crear una atmósfera modificada para el fruto; concluyeron que la cera cumplía su misión como agente retardador de pérdida de peso, pero no ejercía efecto alguno sobre la producción del etileno conforme avanza el ciclo climatérico, de otro lado se encontró un efecto sinérgico entre la cera y el 1-MCP, para reducir la pérdida de dureza, al actuar directamente sobre la poligalacturonasa que es el principal agente causal para este tipo de deterioro.

El 1-MCP es ampliamente usado debido a su muy baja o nula toxicidad, facilidad de aplicación y 
buena eficiencia con una gran cantidad de cultivos hortofrutícolas. Como originalmente describió Serek et al. (1994) una de las mejores formas de aplicarlo es en forma gaseosa, en ambientes cerrados. Recientemente se han desarrollado formulaciones de gran potencia del 1-MCP para su aplicación como líquido o espray. Estas nuevas presentaciones han sido diseñadas para facilitar la aplicación en campo y poscosecha donde no sea factible contar con instalaciones selladas para la aplicación de gas. Choi et al. (2007) demostraron la eficacia de formulaciones líquidas de 1-MCP, para controlar la maduración poscosecha del aguacate 'Hass', donde encontraron que tratando la fruta con soluciones que contienen 625 $\mathrm{mg} \cdot \mathrm{L}^{-1}$ de 1 -MCP durante 1 minuto, se retrasa de manera significativa el proceso de maduración

Por consiguiente, la investigación no se ha concentrado en la eliminación de etileno, sino en la inhibición de su acción mediante el uso de 1-MCP. A pesar de la disponibilidad de varias tecnologías para absorber etileno (degradación catalítica, carbón activado) la mayoría de los sistemas comerciales para el control de este compuesto se basan en sistemas de ventilación adecuados y oxidación de etileno mediante permanganato de potasio. Sin embargo, los sistemas de ventilación no son siempre apropiados en ambientes sellados o herméticos o donde el control específico de etileno es preciso.

Recientemente se ha evaluado la supresión de la maduración en aguacates con un nuevo depurador de etileno provocado por paladio (Terry et al., 2007), el cual presenta una significativa capacidad de adsorción de etileno $\left(4.162 \mathrm{~mL} \cdot \mathrm{g}^{-1}\right.$ de material) a $20^{\circ} \mathrm{C}$ y aproximadamente $100 \% \mathrm{HR}$ que demostró ser muy superior a $\mathrm{KMnO}_{4}$ cuando se utiliza en pequeñas cantidades y bajo condiciones de humedad relativa alta, para este caso la capacidad de adsorción óptima fue calculada aproximadamente como $10,000 \mathrm{~mL} \cdot \mathrm{g}^{-1}$, este material no provocó alteraciones en la consecuente maduración del fruto.

\section{Atmósferas modificadas o controladas}

Es conocido desde hace mucho tiempo que las atmósferas modificadas (AM) ayudan a reducir la pérdida de calidad durante la etapa poscosecha de un amplio rango de frutas (Kader et al., 1989), los primeros trabajos para el aguacate con AM datan de hace una década (Meir et al., 1995, 1997; Yahia et al., 1998). Hertog et al. (2003) evaluaron 32 diferentes $\mathrm{AM}$ a $7^{\circ} \mathrm{C}$ para la conservación de aguacate 'Hass', encontrando que las condiciones óptimas que mantenían la mayoría de atributos de calidad eran $2 \mathrm{kPa} \mathrm{O}_{2}$ y $0 \mathrm{kPa} \mathrm{CO}$, con lo cual se logró una conservación durante 30 días.

El desarrollo de productos de IV Gama (mínimamente procesados), ha permitido mantener hasta por 28 días mitades y pulpa de aguacate en condiciones de refrigeración a $1,0 \pm 0,5^{\circ} \mathrm{C}$, utilizando modificación de atmósferas de $80 \% \mathrm{CO}_{2}-20 \% \mathrm{~N}_{2}$. Los cultivares que mostraron mejor comportamiento fueron: 'Edranol', 'Gwen' y 'Hass' con niveles de madurez sobre 30\% de materia seca (Salinas, 2001, citado por Olaeta, 2003). Esta técnica, aunque no muy difundida en aguacates, permite tener un producto muy similar a la fruta fresca, en relación a su textura, color y sabor, listos para su utilización, lo que reduce tiempos y costos por ese concepto. Es importante señalar que la fruta conservada en estas condiciones presenta un cierto nivel de respiración el que disminuye con el tiempo; después de 30 días de almacenamiento, la fruta pierde rápidamente su calidad, desarrollando cierto grado de pardeamiento y de microorganismos, sin embargo; los niveles que alcanzan estos últimos, son inferiores a los considerados peligrosos para la salud (Verdes et al., 1995).

Esta técnica también ha sido probada a escala comercial (Lemmer et al., 2003), para lo cual contenedores de aguacates 'Hass' y 'Fuerte', se trataron con SmartFresh ${ }^{\circledR}(\mathrm{SF})$ o en atmósfera controlada (AC), la fruta en atmósfera controlada necesitó un promedio de 4,98 días para madurar, mientras que la fruta con SF requirió 5,44 días. 
En ambos cultivares, la fruta de mayor calibre con SF maduró más rápido que la fruta almacenada en AC, mientras que se verificó lo opuesto para los calibres más pequeños. Los resultados permiten recomendar para estas dos variedades en cuanto al contenido de humedad del 70\% (30\% de masa seca) como punto límite para cosechar fruta, que se someta a tratamiento con SF para destinarse al sector del mercado "Maduro y listo para consumir" (en inglés, Ripe and Ready).

\section{Control de bacterias y hongos}

En el campo de la eliminación de daños poscosecha causados por bacterias y hongos, se encuentran reportados estudios (APHIS, 2000) que indican que el uso de radiaciones en bajas dosis, pueden ser usadas para tal fin en frutas frescas; para el caso del aguacate Arevalo et al. (2002) ensayaron el uso de radiaciones gamma (100, 250, $350 \mathrm{~Gy})$, producidas por una fuente de Cobalto 60, en aguacates 'Hass' frescos para prolongar su vida útil a temperatura ambiente, por la eliminación de bacterias que causan deterioro, se analizó el efecto desde el punto de vista histológico, bioquímico y fisiológico; se observaron daños principalmente en el tejido parenquimático, donde las células de la membrana fueron plasmolisadas y se formó un color rojo atribuido al desarrollo de fenoles, a pesar de esto, la evaluación sensorial no detectó cambios significativos y se demuestra que el uso de dosis de $100 \mathrm{~Gy}$ son efectivas para desinfectar el aguacate.

\section{PROCESAMIENTO}

Pulpa. El método de conservación que ha obtenido los mejores resultados corresponde al puré congelado (Soliva, 2002), el cual se puede utilizar como base de productos tipo salsa, para rodajas de papas y galletas saladas, entre otras (Ramtahal et al., 2007). Se puede emplear también como componente del producto denominado "guacamole", muy popular en México y ahora en Estados Unidos, el cual utiliza como base el puré de aguacate, sal, polvo de cebolla y jugo de limón (Carvallo, 1982). Debido a su elevado valor nutritivo, se han intentado numerosas formas de conservación, tales como pastas, las cuales una vez secas, pudieran lograr un polvo estable y aceptable. Sin embargo, debido al alto contenido en lípidos no se ha logrado el producto deseado, el procesamiento para enlatado no ha logrado buenos resultados debido a que la pulpa desarrolla sabores amargos al calentarla (Bower et al., 2003).

Una de las tecnologías más promisorias para la conservación del guacamole es el uso de altas presiones tal como lo demostraron (Lopez et al., 1998; Palou et al., 2000), quienes determinaron que aplicando ciclos de $15 \mathrm{~min}$ a $689 \mathrm{MPa}$, se reduce hasta en un $85 \%$ la actividad de la polifenoloxidasa, sin causar daño en las características sensoriales del producto. La liofilización ha logrado un producto aceptable, posible de ser utilizado como base para otros productos, el polvo puede empacarse en simples bolsas de polietileno o de aluminio laminado con polietileno, y posteriormente rehidratarlo para lograr la consistencia deseada (Arriola et al., 2006).

En relación con el puré congelado la temperatura más recomendada es $-18^{\circ} \mathrm{C}$, los productos así conservados pueden almacenarse durante 8-10 meses, sin embargo su calidad comienza a decrecer después de 3 meses en almacenamiento; otro de los inconvenientes es la significativa pérdida de textura que ocurre después de la descongelación, debido a la destrucción celular. Para contrarrestar este fenómeno, algunas investigaciones sugieren la eliminación del agua antes de congelar el producto, por medio de baños en agua caliente a $80^{\circ} \mathrm{C}$ (Valenzuela, 1996; Bower et al., 2005).

En todos estos productos cobra especial importancia el control del pardeamiento enzimático, el cual debe realizarse utilizando antioxidantes ya que el uso de altas temperaturas afecta la calidad de la pulpa (Ortiz et al., 2003). Los antioxidantes más utilizados para mantener las condiciones de color son principalmente: el bisulfito de sodio, el 
ácido ascórbico, ácido isoascórbico y ácido cítrico, algunas pulpas que se comercializan en la actualidad, presentan un elevado nivel de aditivos estabilizantes como son: gomas, alginatos, polifosfatos y otros que reducen el desarrollo microbiano, como son el sorbato y benzoato de sodio o potasio (Gómez, 2002).

En conjunto, estos aditivos pueden alcanzar niveles superiores a $20 \%$, lo que reduce el porcentaje de aguacate en la mezcla, bajando la calidad del producto final. El uso de aditivos en las pulpas deberá reducirse paulatinamente, ya que la tendencia de los consumidores es consumir cada vez más productos sin aditivos o con un nivel bajo de ellos, una buena pulpa no debe contener menos de un 98 o 99\% de aguacate (Elez et al., 2005).

El uso de esta técnica de conservación, requiere utilizar envases que tengan una baja permeabilidad al $\mathrm{N}_{2}$ y al $\mathrm{CO}_{2}$ así como al $\mathrm{O}_{2}$. El uso de polietileno de baja densidad y plásticos aluminizados ha mostrado tener un buen comportamiento en este tipo de productos. Se ha determinado también que aguacates combinados con lechugas logran un producto de IV Gamma aceptable para el consumidor hasta por un período de 15 días, almacenadas a $1^{\circ} \mathrm{C}$ y con modificación atmosférica de $\mathrm{CO}_{2}$ y $\mathrm{N}_{2}$ (Ormazábal, 1999).

Además de los productos mencionados, se han desarrollado otros como la deshidratación osmótica, utilizando maltodextrinas $\mathrm{y} \mathrm{NaCl}$, que ha logrado mantener el aguacate estable en relación al color y la actividad microbiológica (Schwartz et al., 2001a). También se ha obtenido polvo de aguacate, secado por atomización, la que presentó buena estabilidad en relación al color y actividad microbiana, sin embargo, será necesario estudiar más estrictamente los tiempos y temperaturas del proceso, ya que el desarrollo de sabores extraños reduce la calidad del producto. (Schwartz et al., 2007).

La inhibición del pardeamiento enzimático causado por la polifenoloxidasa (PPO), por medio de técnicas combinadas (adición de EDTA y atmósfera rica en $\mathrm{N}_{2}$ ), y evaluada en términos de la actividad de la PPO y los parámetros de color CIELab, los purés no tratados mostraron una rápida disminución en la luminosidad y nivel de color verde, el EDTA afectó el color inicial de la pulpa, pero fue el más efectivo controlando el pardeamiento (Soliva et al., 2001).

El efecto de métodos combinados sobre la preservación de la microflora natural presente en el puré de aguacate, ha sido estudiado evaluando el efecto de la adición de ácido ascórbico, modificación de la actividad de agua, reducción del pH, cambio en la atmósfera de empaque y el control de la temperatura de almacenamiento, los resultados mostraron que la adición de $300 \mathrm{mg} \cdot \mathrm{kg}^{-1}$ de ácido ascórbico puede extender la estabilidad del puré por períodos superiores a 4 meses. El empaque al vacío con almacenamiento a $4^{\circ} \mathrm{C}$, mostró una alta influencia sobre el nivel poblacional de los microorganismos presentes, conservándole por 112 días, sin adición de antimicrobianos por último; la adición de maltosa para reducir la actividad de agua hasta 0,96 condujo a una mejor preservación del puré, pero produjo cambios sustanciales en la palatabilidad del producto (Soliva, 2004).

Valle-Guadarrama (2005) y su grupo han estudiado el coeficiente de difusión másico, para el oxígeno, en tejidos de frutos de aguacate variedad 'Hass', evaluados a $20^{\circ} \mathrm{C}$, desarrollando un modelo de respiración-difusión, usando una rutina para balance de masa en estado no estacionario, en combinación con la segunda ley de Fick y la teoría cinética de Michaelis-Menten. El resultado arrojado fue de $2,2 \times 10^{-9} \cdot \mathrm{m}^{-2} \cdot \mathrm{s}^{-1}$.

Aceite. El aguacate, dependiendo de la variedad y madurez, alcanza en la pulpa niveles de hasta $25 \%$ de aceite, con valores promedios de $15-19 \%$, lo que permite lograr rendimientos de alrededor de 10\% de la fruta fresca (Schwartz et al., 2001b). La composición del aceite crudo de aguacate contiene alrededor de un 80-85\% de ácidos grasos insaturados así como un importante nivel de 
materia insaponificable (Ortega, 2003). La propiedad de ser un fruto con un elevado nivel de aceite, junto a otras, como son su porcentaje de proteínas, hidratos de carbono, vitaminas y minerales, le otorgan un gran valor nutritivo. Además, posee propiedades notables sobre compuestos como el colesterol, beneficiando, por tanto, la salud humana (Valenzuela, 1996).

Se han probado una serie de métodos para la extracción del aceite de frutos de aguacate, donde el objetivo primordial siempre ha sido el obtener el mayor rendimiento sin dañar su calidad, la extracción por solvente puede dar los mejores resultados, pero resulta demasiado costoso y peligroso de utilizar por su inflamabilidad; además, la remoción de todo el solvente desde el aceite es difícil y pequeñas trazas pueden causar detrimento de la calidad de dicho aceite (Human, 1997). La industria farmacéutica francesa considera que la fracción insaponificable del aceite debe ser recuperada desde el aceite de aguacate debido al gran valor que representa, ya que contiene un esterol llamado fitosterol (Duester, 2001), que posee las mismas habilidades que la lanolina. Esta particularidad es muy apropiada para la piel y cremas de masajes.

La propiedad de penetración en la piel es la clave del éxito de este aceite como una ayuda natural y efectiva. Además, la calidad del aceite es ideal para adicionar otras sustancias incapaces de penetrar por sí solas a la piel, también el aceite se utiliza en jabones de baño ya que ayuda a aumentar la espumosidad y las cualidades de limpieza, es fácil de emulsificar y su baja tensión superficial produce cremas y jabones suaves (Pérez et al., 2005).

El rico contenido vitamínico del aceite de aguacate beneficia a la industria de la cosmética ya que la vitamina A ayuda a prevenir la sequedad de la piel y la vitamina $\mathrm{E}$ (tocoferol) junto a la $\mathrm{D}$, son efectivas contra el envejecimiento de la piel, debido a la abundancia de ácidos grasos insaturados. Las proteínas fibrosas (cercanas al 4\%) ac- túan como un humectante natural de la piel. Por último, el aceite de aguacate posee propiedades de bronceador, retardando el envejecimiento ocasionado por los rayos UV (Cervantes, 2007).

Turatti et al. (1985) encontraron que cuando la pulpa de aguacate se deshidrata y es sujeta a presión hidráulica, se obtiene el aceite con el mayor porcentaje de materia insaponificable $(4,9 \%)$ y también de tocoferoles (6,92\%). Desde la fracción insaponificable, Joseph y Neeman (2002) aislaron compuestos polialcohólicos, sólo presentes en aceite de aguacate, importante en propiedades cosméticas, por aislamiento de estos compuestos pueden detectarse adulteraciones en el aceite. El aceite de aguacate, al tratarse con temperatura, presenta como desventaja el rápido desarrollo de un sabor amargo y son varios los compuestos responsables de la astringencia, de acuerdo con esto, es extremadamente difícil prevenir o modificar la inducción calórica del mal sabor para permitir un proceso térmico exitoso (Jiménez et al., 2001).

Actualmente la tendencia es a utilizar en forma creciente el aceite de aguacate para uso culinario, por tener entre otras cualidades un alto punto de humo, excelente sabor y un color verde muy atractivo, siendo preferido por los chef de cocina de la alta gastronomía europea, sustituyendo al aceite de oliva. Eyres (2001), comparó el aceite de oliva con el aceite de aguacate, determinando que este último presenta mayores niveles de clorofila, índice de yodo, vitamina $\mathrm{E}$ y un menor nivel de acidez libre, lo que constituye una mejor calidad. En Nueva Zelanda, Sudáfrica, México y otros países, se ha generado una serie de grupos de trabajo que han centrado su esfuerzo en la extracción de aceite de aguacate "extra virgen", el cual se obtiene a partir del fruto sin piel y sin semilla, con un tratamiento de macerado en frío, y luego filtrado y embotellado (Requejo et al., 2007).

Con ello, el aceite mantiene todas las propiedades originales que lo hacen ser muy aceptable para la salud humana, ya que al no contener colesterol previene enfermedades cardíacas, reduciendo in- 
cluso problemas de próstata. También este aceite "extra virgen" de aguacate se está comercializando en cápsulas lo que lo hace ser un buen suplemento dietético y como aerosol para cocinar o condimento (Avocado Oil Nz Ltd., 2007). El aceite de aguacate se deteriora durante el almacenaje, por lo que la temperatura de almacenaje no debe ser superior a $25^{\circ} \mathrm{C}$, y el tipo de envase oscuro, para preservar por más tiempo la calidad del producto (Alicia et al., 2004).

Semilla. Por medio de observaciones microscópicas de la semilla de aguacate se encontró que las células del parénquima de cotiledones almacenan la mayor cantidad de almidón, mientras que en el embrión se almacena la mayor parte de la grasa. Respecto a las extracciones de la grasa de la semilla de aguacate, se han ensayado técnicas convencionales y extracción mediante fluido supercrítico, usando hexano y dióxido de carbono. En estas condiciones se ha extraído aproximadamente la misma cantidad de grasa, 3,08 y $3,07 \%$ respectivamente, mientras que con etanol se extrajo el 0,79\% (García, 1999).

Saldarriaga et al. (2002) realizaron la extracción del colorante de la semilla del aguacate, controlando variables de proceso como concentración de la solución, acidez $(\mathrm{pH})$, tiempo de la extracción, relación sólido / solvente y temperatura del proceso. La optimización se realizó con la técnica de superficies de respuesta, el colorante obtenido se caracterizó por espectro IR, UV-VIS, TLC, el producto generado mostró gran potencial para ser usado en el área textil y de artesanías.

\section{CONCLUSIONES}

El mercado mundial hortofrutícola se caracteriza por ser altamente competido desde el punto de vista de la oferta, por tener una creciente concentración de empresas transnacionales que dominan los canales internacionales del comercio y por la importancia de las cadenas de supermercados y tiendas especializadas en la venta minorista. Es muy exigente en cuanto a los requisitos de ingreso a los mercados de destino, tanto en normas sanitarias como de calidad y de presentación de los productos. Existe una gran cantidad de literatura referente a la cosecha y poscosecha del aguacate, lamentablemente esta información no se transfiere de manera oportuna a los productores y comercializadores, para que coloquen en práctica los avances tecnológicos que se generan, limitando con esto la competitividad del producto fresco, en todos los niveles de la cadena productiva.

El tema de la agroindustrialización y procesamiento ha sido limitado de forma muy marcada a la investigación sobre métodos de conservación combinados, aplicados a la pulpa, en la mayoría de los casos no se analiza la aplicabilidad comercial y sólo se investiga aisladamente a nivel de laboratorio. En Colombia, el consumo en fresco demanda la mayor parte de la producción nacional, la agroindustria se limita a pequeñas producciones de puré mezcladas con diferentes especias, que se comercializan a nivel de pequeños restaurantes; la falta de conocimiento sobre las características específicas de las variedades sembradas, no permite definir claramente el procesamiento más adecuado para cada una de estas.

Son muchos los productores y consumidores que quieren pagar cada vez menos por productos básicos. Aun así, para seguir conquistando los mercados externos y mantener el consumo local se les debe agregar valor o al menos algo que nos permita mantenerlos, y donde el principal atributo es la confiabilidad, la marca y la creatividad.

Los desafíos a futuro, para el sector del aguacate y también esta oportunidad de negocio no son la excepción, las oportunidades son infinitas, las ventajas radican en incrementar el consumo y poder entrar en mercados en los cuales hoy los productos convencionales no son competitivos, buscando eliminar las debilidades de la fruta fresca y no competir directamente con los grandes productores de otros países, sino ofrecer un producto con gran diferenciación. 


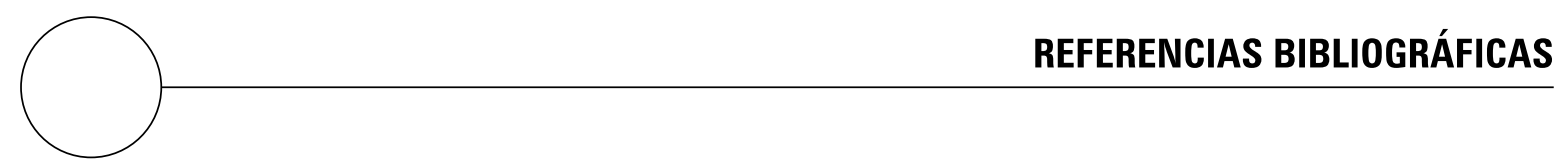

Agexpront. 2004. Estudio de oportunidad de negocios sobre el aguacate. Departamento de información de mercados. Guatemala. 34 p.

Aphis. Animal and plant health inspection service, USDA, 2004. Importation of avocado fruit (Persea americana Mill. var. 'Hass') from Mexico. A Risk Assessment. pp. 141 p.

Aphis. Animal and plant health inspection service, USDA. 2000. Irradiation as a phytosanitary treatment of imported fruits and vegetables. Proposed rule. Fed. Reg. 67(205), 65016-65029.

Arevalo, L.; M. Bustos y C. Saucedo. 2002. Changes in the vascular tissue of fresh Hass avocados treated with cobalt 60. Radiat. Phys. Chem. 63, 375-377.

Arpaia, M.L.; Z. van Rooyen; P.J. Hofman y A.B. Woolf. 2004. Las prácticas culturales influyen sobre la calidad de la fruta en postcosecha. $2^{\circ}$ seminario internacional de paltos. Quillota, Chile. 1-10.

Arriola E.; T. García; G.M. Guatemala y J. Nungaray Arellano. 2006. Comportamiento del aguacate Hass liofilizado durante la operación de rehidratación. Revista Mexicana de Ingeniería Química 5(1), 51-56.

Asohofrucol (Asociación Hortifrutícola de Colombia). 2006. Plan Frutícola Nacional. Desarrollo de la Fruticultura en Tolima. Cali. 78 p.

Avilán, L.; M. Rodríguez; R. Carreño e I. Dorantes. 1994. Selección de variedades de aguacate. Agronomía Tropical 44(4), 593-618.

Avocado Oil Nz Ltd. 2007. Product information. The Grove. Disponible online en: http://www.avocado-oilco. nz/products.html; consulta: diciembre de 2007.

Bower, J.P. y M.T. Dennison. 2003. Progress in the development of avocado products. South African Avocado Growers' Association Yearbook 26, 35-37.

Bower, J.P. y M.T. Dennison. 2005. A process to prevent browning of frozen avocado halves and chunks. South African Avocado Growers' Association Yearbook $28,40-41$.

Bravo, O.M. 1997. Efecto de la época de cosecha y la temperatura de almacenaje en la calidad de frutos de palto (Persea americana Mill.) cv. Gwen. Trabajo de grado. Universidad de Chile, Santiago. 69 p.
Cervantes, M. 2007. Catálogo de propiedades nutrimentales, nutracéuticas y medicinales del aguacate. Secretaría de Desarrollo Rural del Estado de Puebla. México. 11 p.

Choi, S.T.; P.T. Souvaltzis; C. Lim y D.J. Huber. 2007. Suppression of ripening and induction of asynchronous ripening in tomato and avocado fruits subjected to complete or partial exposure to aqueous solutions of 1-methylcyclopropene. Postharvest Biol. Technol. (en imprenta).

Duester, K. 2001. Avocado fruit is a rich source of BetaFitosterol. J. Amer. Diet. Assoc. 101(4), 404-405.

Everett, K.R.; I.C. Hallett; J.R. George; R.W. Chynoweth y H.A. Pak. 2007. Avocado lenticel damage: The cause and the effect on fruit quality. Postharvest Biol. Technol. (en imprenta).

Eyres, L.; N. Sherpa y G.Hendriks. 2001. Avocado oil: A new edible oil from australasia. Disponible online en: http://www.olivado.com/studies4.htm; consulta: diciembre de 2007.

Feng, X.; A. Apelbaum; E.C. Sisler y R. Goren, 2000. Control of ethylene responses in avocado fruit with 1 methylcyclopropene. Postharvest Biol. Technol. 20, 143-150.

García, J.A. 1999. Estructura de la semilla de aguacate y cuantificación de la grasa extraída por diferentes técnicas. Revista Chapingo Serie Horticultura 5, 123-128.

Gerdes, D.L. y V. Parrino-Lowe, 1995. Modified atmosphere packaging (MAP) of Fuerte avocado halves. Food Sci. Technol.-Leb. 28, 12-16.

Gómez López, V.M. 2002. Inhibition of surface browning, cut avocado. J. Food Quality 25, 369-379.

Hertog, M.; S.E. Nicholson y K.Whitmore. 2003. The effect of modified atmospheres on the rate of quality change in 'Hass' avocado. Postharvest Biol. Technol. 29, 41-/53.

Human, T.R. 1997. Oil as byproduct of the avocado. South African Avocado Growers' Association Yearbook 10, 163-164.

Instituto Colombiano de Normas Técnicas y Certificación 1994. Productos agrícolas. Aguacate. ICONTEC, Bogotá. 6 p. 
Instituto Colombiano de Normas Técnicas y Certificación. 1996. Frutas frescas. Aguacate. Almacenamiento y transporte. ICONTEC, Bogotá. 10 p.

Instituto Colombiano de Normas Técnicas y Certificación. 2003. Frutas frescas. Aguacate. Variedades mejoradas. Especificaciones. ICONTEC, Bogotá. 24 p.

Jeong, J.; D.J. Huber y S.A. Sargent. 2002. Influence of 1 - methylcyclopropene (1-MCP) on ripening and cell wall matrix polysaccharides of avocado. Postharvest Biol. Technol. 25, 241-256.

Jeong, J.; D.J. Huber y S.A. Sargent. 2003. Delay of avocado (Persea americana) fruit ripening by 1-methylcyclopropene and wax treatments. Postharvest Biol. Technol. 28, 247-257.

Jiménez, M.H.; M.R. Aguilar; M.L. Zambrano y E. Kolar. 2001. Propiedades físicas y químicas del aceite de aguacate obtenido de puré deshidratado por microondas. Revista de la Sociedad Química de México 45(2), 89-92.

Joseph, D. y I. Neeman. 2002. Characterization of avocado oil by polyalchoholic compounds in the unsaponifiable fraction. Dept. of Food Eng. Biotech. Technicon, Israel. Ins. of Tech. Hiafa, Israel. Ivista Italiana delle Sostage Grasse 59(6), 279-284.

Kader, A.A.; D. Zagory y E.L. Kerbel. 1989. Modified atmosphere packaging of fruits and vegetables. Crit. Rev. Food Sci. Nutr. 28, 1-30.

Lemmer, D.; J. Bezuidenhout; S. Sekhune; P. Ramokone y L. Letsoalo. 2003. Semi-commercial evaluation of SmartFresh with South African export avocados in static containers at a packinghouse during 2002. Memorias $\mathrm{V}$ congreso internacional del aguacate. pp. 617-622.

López-Malo, A.; E. Palou; G.V. Barbosa-Canovas y J. Welti-Chanes. 1998. Polyphenoloxidase activity and color changes during storage of high hydrostatic pressure treated avocado puree. Food Res. Int. 31(8), 549-556.

Martínez, P.; R. Soliva; S. Gorinstein y O. Martín. 2005. Natural antioxidants preserve the lipid oxidative stability of minimally processed avocado purée. J. Food. Sci. 70(5), 325-329.

Matthew, F.; P. Adkins; J. Hofmanb; B. Stubbings y A. Macnish. 2005. Manipulating avocado fruit ripening with 1-methylcyclopropene. Postharvest. Biol. Technol. 35, 33-42.

Meir, S. Y. Akerman; M. Fuchs y G. Zauberman. 1995. Further studies on the controlled atmosphere storage of avocados. Postharvest Biol. Technol. 5, 323-330.
Meir, S.; D. Naiman; M. Akerman; J.Y. Hyman; G. Zauberman y Y. Fuchs. 1997. Prolonged storage of 'Hass' avocado fruit using modified atmosphere packaging. Postharvest Biol. Technol. 12, 51-/60.

Olaeta, J.A. 2003. Industrialización del aguacate: Estado actual y perspectivas futuras. Actas V Congreso Mundial del Aguacate. pp. 749-754.

Ormazábal, P.A. 1999. Efecto de la IV Gama en la mezcla de lechuga (Lactuca sativa) tipo escarola y aguacate (Persea americana Mill.) cvs. Edranol Hass y Negra de La Cruz. Trabajo de grado. Facultad de Agronomía. Universidad Católica de Valparaíso.

Ortega, M. 2003. Valor nutrimental de la pulpa fresca de aguacate Hass. Memorias V Congreso Mundial del Aguacate. pp. 741-748.

Ortiz, A.; R. Mora; T. Santiago y L. Dorantes. 2003. Obtención de una pasta de aguacate mediante tratamiento térmico. Memorias V Congreso Mundial del Aguacate. pp. 761-768.

Ortiz, M.; A. Dorantes; M. Galndez y S. Cárdenas. 2004. Effect of a novel oil extraction method on avocado (Persea americana Mill.) pulp microstructure. Plant Food. Hum. Nutr. 59, 11-14.

Palou, E.; C. Hernandez; A. Lopez; G.V. Barbosa-Canovas; B.G. Swansonc y J. Welti-Chanes. 2000. High pressure-processed guacamole. Innovative Food Sci. \& Emerging Technol. 1, 69-75.

Pérez Rosales, R.; S. Villanueva Rodríguez y R. Cosio Ramírez. 2005. El aceite de aguacate y sus propiedades nutricionales. En: http://redalyc.uaemex.mx/redalyc/ pdf/730/73000310.pdf. consulta: diciembre de 2007

Pesis, E.; M. Ackerman; R. Ben-Arie; O. Feygenberg; X. Akiva; R. Goren y D. Prusky. 2002. Ethylene involvement in chilling injury symptoms of avocado during cold storage. Postharvest Biol. Technol. 24, 171-181.

Ramtahal, G.A.; J.O. Akingbala y G. Baccus. 2007. Laboratory preparation and evaluation of Pollock variety avocado (Persea americana Mill.) guacamole. J. Sci. Food Agric. 87, 2068-2074.

Requejo, C.; S. Olsson; T. McGhie; L. Eyres; O. Ashton; Y. Wang; M. Wong y A.Woolf. 2007. Aceite de aguacate prensado en frío - factores previos y posteriores a la cosecha que influencian su calidad. En: Avocadosource. http://www.avocadosource.com/WAC6/es/ Resumen/4b-194.pdf. consulta: diciembre de 2007.

Rojas, J.M.; A.E. Peñuela; C. Rocio; G.E. Aristizabal y M.C. Chaparro. 2004. Caracterización de los productos hortofrutícolas colombianos y establecimiento de las normas técnicas de calidad. Cenicafe, Chinchina. pp. 163-178. 
Saldarriaga, D.F. y J.E. Devia Pineda. 2002. Diseño de un proceso de extracción industrial del colorante de la semilla del aguacate. Trabajo de grado. Universidad EAFIT, Medellín. pp. 36-43.

Saltveit, M.E. 1999. Effect of ethylene on quality of fresh fruits and vegetables. Postharvest Biol. Technol. 15, 279-292.

Schwartz, M.; J.A. Olaeta; P. Undurraga y M. Sepúlveda. 2007. Obtención y almacenamiento de palta (aguacate) en polvo. Memorias VI congreso mundial del aguacate. Chile. 8 p.

Schwartz, M.; J.A. Olaeta; P. Undurraga y V. Costa. 2001b. Extracción enzimática y caracterización del aceite de palta (Persea americana Mill.). Trabajo de grado. Universidad de Chile, Santiago, Chile: 8 p. En: http:// www.avocadosource.com/WAC6/es/Extenso/4b-182. pdf. consulta: diciembre de 2007.

Schwartz, M.; J.A. Olaeta; P. Undurraga; M. Sepúlveda y P. Tepper. 2001a. Secado de aguacate (Persea americana Mill.) por ósmosis con maltodextrinas y $\mathrm{NaCl}$. Actas III Congreso mundial aguacate. México. 9 p.

Serek, M.; E.C. Sisler y M.S. Reid. 1994. Novel gaseous ethylene binding inhibitor prevents ethylene effects in potted flowering plants. J. Amer. Soc. Hort. Sci. $119,1230-1233$.

Seymour, G.B. y G.A. Tucker. 1993. Avocado. En: Seymour, G.B.; J. Tayler y G.A. Tucker (eds.). Biochemistry of fruit ripening. Chapman \& Hall, London. pp. 53-81.

Soliva, R.; P. Elez; M. Sebastián y O. Martín. 2001. Evaluation of browning effect on avocado puree preserved by combined methods. Innovative Food Sci. Emerging Technol. 1, 261-268.

Soliva-Fortuny, R. y Elez-Mart, P. 2004. Effect of combined methods of preservation on the naturally occurring microflora of avocado puree. Food Control 15, 11-17.

Soliva-Fortuny, R.C.; P. Elez-Martnez; M. Sebastian-Caldero y O. Martn-Belloso. 2002. Kinetics of polyphenoloxidase activity inhibition and browning of avocado puree preserved by combined methods. J. Food Eng. 55(2), 131-137.

Terry, L.A.; T. Ilkenhans; S. Poulston; L. Rowsell y A.W.J. Smith. 2007. Development of a new palladium-promoted ethylene scavenger. Postharvest Biol. Technol. 45, 214-220.
Today's Market Prices. 2003. Aguacate fresco. Panorama mundial e informe de mercado y precios de Estados Unidos. En: Serie Estudios de Mercado N 1/03.38.

Turatti, J.M.; L.C. Santos y K.K. dos Tanqojs \& Arima. 1985. Characterisation of avocado oil extracted by various methods. Ins. de Technología de Alimentos, Compinas, Sao Paulo, Brazil. Oletim do Institute de Technologia de Alimentos Brazil 22(2), 267-284.

Valenzuela Reyes, R.E. 1996. Evaluación de congelado en palta (Persea americana Mill), en los cultivares Fuerte, Hass, Edranol y Bacon bajo distintas formulaciones. Trabajo de grado. Universidad Católica de Valparaíso, Quillota, Chile. 84 p.

Valenzuela, A.M. 1996. Extracción y caracterización del aceite de palta. Trabajo de grado. Facultad Ingeniería, Universidad Católica de Valparaíso. Valparaíso. $106 \mathrm{p}$.

Valle-Guadarrama, S. y T. Espinosa-Solares. 2005. Oxygen diffusivity in avocado fruit tissue. Biosystems Engineering 92 (2), 197-206.

Watkins C.B. 2006. The use of 1-methylcyclopropene (1$\mathrm{MCP})$ on fruits and vegetables. Biotechnol. Adv. 24 , 389-409.

Woolf, A.; J. Bowena.; S. Ball; S. Durand; W.G. Laidlawb y I.B. Ferguson. 2004. A delay between a $38^{\circ} \mathrm{C}$ pretreatment and damaging high and low temperature treatments influences pretreatment efficacy in 'Hass' avocados. Postharvest Biol. Technol. 34, 143-153.

Woolf, A.B.; A. Wexler; D. Prusky; E. Kobiler y S. Lurie. 2000. Direct sunlight influences post-harvest temperature responses and ripening of five avocado cultivars. J. Amer. Soc Hort. Sci. 125(3), 370-376.

Woolf, A.B.; C.B. Watkins; J.H. Bowen,; M. Lay-Yee; J.H. Maindonald, y I.B. Ferguson, 1995: Reducing external chilling injury in stored 'Hass' avocados with dry heat treatments. J. Amer. Soc. Hort. Sci. 120(6), 1050-1056.

Woolf, A.B.; J.H. Bowen y I.B. Ferguson. 1999. Preharvest exposure to the sun influences post-harvest responses of 'Hass' avocado fruit. Postharvest Biol. Technol. 15(2), 143-153.

Yahia, E. y G. Gonzalez. 1998. Use of passive and semiactive atmospheres to prolong the postharvest life of avocado fruit. Lebensm. Wiss. Technol. 31, 602-606. 